\title{
Biosimilar Epoetin Zeta in Nephrology: Effect of Injection Frequency on Weekly Dose
}

\section{Gerhard Lonnemann, Eike Wrenger}

Outpatient Clinic of Nephrology, Hemodialysis Center, Eickenhof, Langenhagen, Germany.

Email: lonnemann@eickenhof-dialyse.de

Received September $27^{\text {th }}, 2012$; revised October $14^{\text {th }}, 2012$; accepted November $7^{\text {th }}, 2012$

\begin{abstract}
Aim: This observational clinical study tested the effect of injection frequency of the biosimilar epoetin zeta on the weekly dose needed to maintain stable hemoglobin levels in chronic kidney diseases (CKD) patients on intermittent high-flux hemodialysis (HD). Patients and Methods: CKD patients $(n=33)$ on regular HD therapy 3 times a week were treated for 18 months with epoetin zeta i.v. The hemoglobin levels, the weekly dose as well as the injection frequency of epoetin zeta were monitored at least every two weeks. Patients were followed in three time periods: 1) extended follow-up (months 1 - 18); 2) intervention phase (months 19 - 21); and 3) post intervention observation phase (months 28 - 30). During extended follow-up the majority of patients $(n=21)$ received only one injection of epoetin zeta per week. During the intervention phase, injection frequency was increased to 3 injections per week in all patients accompanied by a reduction in weekly doses of approximately $20 \%-30 \%$. Following a 9-month period of dose adjustment all parameters were monitored again in the post-intervention phase. Results: During the first 18 months of epoetin zeta therapy the mean hemoglobin level was stable between 11 and $12 \mathrm{~g} / \mathrm{dl}$. The mean weekly dose of epoetin zeta was $7939 \mathrm{IU} /$ week in month 6 and $7909 \mathrm{IU} /$ week in month $18(\mathrm{p}=$ not significant). The mean frequency (injections/week) was 1.27 in month 6 and 1.29 in month 18 (not significant). Compared to month 18, at the end of the observation at month 30, hemoglobin levels were stable, mean injection frequency increased to $2.25(\mathrm{p}<0.001)$ and the mean weekly dose decreased to $5469 \mathrm{IU} /$ week $(-31.7 \%, \mathrm{p}<0.001)$. Conclusions: Increasing the injection frequency of the short acting biosimilar epoetin zeta to two to three injections per week reduces the weekly dose and thereby the costs of ESA therapy of renal anemia significantly.
\end{abstract}

Keywords: Epoetin Zeta; Renal Anemia; Drug Efficiency; Injection Frequency; Chronic Hemodialysis

\section{Introduction}

The biosimilar epoetin zeta has recently been introduced as a new drug to treat patients with chronic kidney diseases (CKD) and renal anemia. First studies proved a high degree of similarity of epoetin zeta with epoetin alfa leading to approval of epoetin zeta to be biosimilar to epoetin alfa by the EMEA [1-4]. Epoetin zeta is a shortacting erythropoiesis-stimulating agent (ESA) and is recommended to be used with a frequency of 3 injections per week either intravenously or subcutaneously.

In our first clinical observational study CKD patients on intermittent hemodialysis $(\mathrm{n}=18)$ were switched from established ESAs ( $80 \%$ darbepoetin alfa) to epoetin zeta and followed for 6 months [5]. In monthly controls, mean hemoglobin levels were maintained stable at 11 $12 \mathrm{~g} / \mathrm{dl}$ throughout the follow-up period, but the weekly dose (using the converting factor: $1 \mu \mathrm{g}$ darbepoetin alfa $=$ $200 \mathrm{IU}$ epoetin zeta) tended to increase by up to $25 \%$ (p $=0.16$, non significant). A surprising result of this small study was that the injection frequency which was one per week or even one in two weeks with the long-acting darbepoetin alfa could be kept in most of the patients after the switch to the short-acting epoetin zeta [5]. The price for keeping the injection frequency low was a slight increase in the weekly dose which was non-significant in our study but may be higher and significant in the hands of other investigators $[6,7]$.

In the present study we tested the effect of injection frequency of intravenously administered epoetin zeta on the weekly dose of this biosimilar ESA.

\section{Patients and Methods}

\subsection{Study Design}

Stable CKD patients ( $\mathrm{n}=33 ; 15$ women, 18 men) on intermittent high-flux hemodialysis (three times a week for 4 - 5 hours each) were enrolled in the study after having given informed consent. The mean age (range) at the 
start of the study was $68.9(38-83)$ years and the time on intermittent hemodialysis was 45.3 (1 - 156) months. The causes of end-stage renal diseases were chronic glomerulonephritis $(\mathrm{n}=11)$, vascular nephropathy/nephrosclerosis $(n=13)$, interstitial nephritis $(n=3)$, diabetic nephropathy $(\mathrm{n}=4)$, polycystic kidney disease $(\mathrm{n}=1)$ and unknown $(\mathrm{n}=1)$. After the switch from established ESAs (82\% darbepoetin alpha) to epoetin zeta all patients were followed for three consecutive time periods: 1) extended follow-up period (months 1 - 18); 2) intervention period (months 19 - 21); and 3) post-intervention observation period (months 28 - 30). Hemoglobin levels, the weekly dose and the injection frequency of the ESAs were documented at least twice a month during the run-in phase of 2 months and during the 18 months of follow-up. During follow-up, the majority of patients $(n=21,64 \%)$ received only one injection of epoetin zeta per week, only two patients received 3 injections per week. During the intervention phase(months 19 - 21) the frequency of epoetin zeta injections was increased to 3 per week in all patients accompanied by a moderate reduction in weekly dose. For example, a patient receiving $1 \times 8000 \mathrm{IU}$ per week was switched to $3 \times 2000$ IU per week, an other from $1 \times 4000$ IU to $3 \times 1000$ IU per week, or a further patient from $2 \times 8000$ IU to $3 \times 4000$ IU per week. Following these three months of adaptation of both frequency and dose, in case of stable hemoglobin $>12 \mathrm{~g} / \mathrm{dl}$ it was allowed to skip a dose and go back to 2 injections per week. Follow-up was extended to 30 months and three time periods were compared: months -2 to 0 (run-in), months 16 - 18 (low frequency) and months 28 - 30 (high frequency). Parameters measured are frequency of injections of epoetin zeta, hemoglobin $(\mathrm{g} / \mathrm{dl})$ and weekly dose (IU/week as well as IU/kg body weight/week).

\subsection{Statistics}

To describe statistically significant changes, the two sided Student's T-test was applied using paired observations. The level of significance was set at $p<0.05$.

\section{Results}

The mean hemoglobin levels were kept very stable between 11 and $12 \mathrm{~g} / \mathrm{dl}$ throughout the extended follow-up period of 18 months under the treatment with epoetin zeta (Figure 1).

Also, the weekly dose of epoetin zeta needed to keep the hemoglobin leves stable did not change significantly during the follow-up period. The mean weekly doses (Figure 2) were at month 0: $7121 \mathrm{IU} /$ week, at month 6: $7939 \mathrm{IU} /$ week and at month 18: $7909 \mathrm{IU} /$ week $(\mathrm{p}=$ not significant).

The frequency of ESA injections per week is depicted in Figure 3. During the run-in phase of the study when

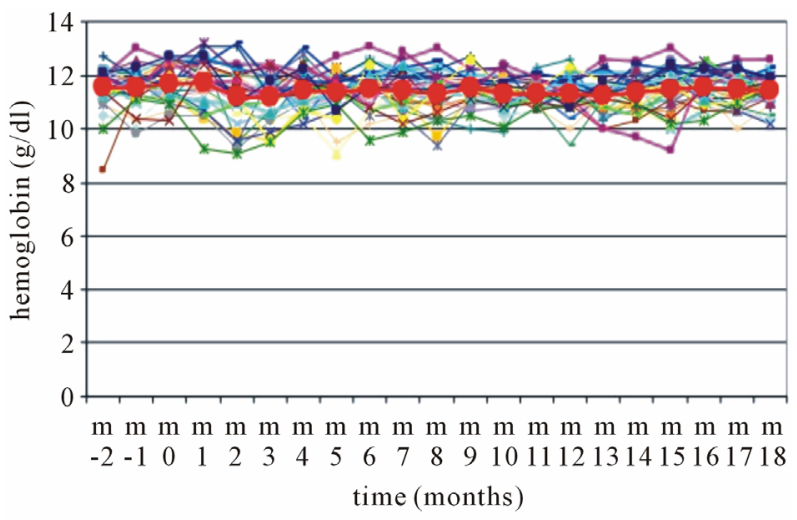

Figure 1. Hemoglobin levels during extended follow-up of epoetin zeta therapy for 18 months. " $\mathrm{m}$ " = month, Epoetin zeta was started in " $\mathrm{m} 0$ ", the bold symbols represent the mean values of $\mathbf{n}=33$ patients.

$82 \%$ of the patients were treated with the long acting darbepoetin alpha, the mean numbers of injections per week were between 0.89 and 1.08 , indicating that the majority of patients received 1 injection per week or even only 1 injection in two weeks (see the bars at the level of 0.5 injections/week in Figure 3). During 18 months of epoetin zeta therapy the mean injection frequency was never higher than 1.58 in month 13 when 4 out of 33 patients received 3 injections per week. The majority of patients $(n=17)$ was treated with 0.5 to 1 injections per week.

At the end of the extended follow-up period of one and a half years, the mean injection frequency was 1.29 injections/week (Figure 4).

Following the study protocol, during months $19-21$ all patients on epoetin zeta were switched to three injections per week accompanied by an average dose reduction of about $25 \%$ per week. This reduction was accomplished by e.g. switching from $1 \times 8000$ IE to $3 \times 2000$ IE per week, or from $1 \times 4000$ IE to $3 \times 1000$ IE per week. Monitoring hemoglobin levels every 2 weeks made it easy to strictly keep the target level of $11-12$ $\mathrm{g} / \mathrm{dl}$ and to adjust the weekly dose of epoetin zeta. The protocol allowed to reduce the injection frequency to 2 injections per week when the hemoglobin level rose above $12 \mathrm{~g} / \mathrm{dl}$. After another 6 months of dose and frequency adjustment, frequency was documented during months 28 - 30 (Figure 4). The mean frequency of epoetin zeta injections was 2.59 in month 28 and decreased to 2.25 in month 30 , when $\mathrm{n}=14$ patients received 3 injections per week, and $n=13$ patients 2 injections per week.

Comparing the mean hemoglobin levels in months 16 18 (low injection frequency) to months 28 - 30 (high injection frequency) hemoglobin levels remained stable within the tide margins (data not shown).

However, with the high injection frequency, the mean 


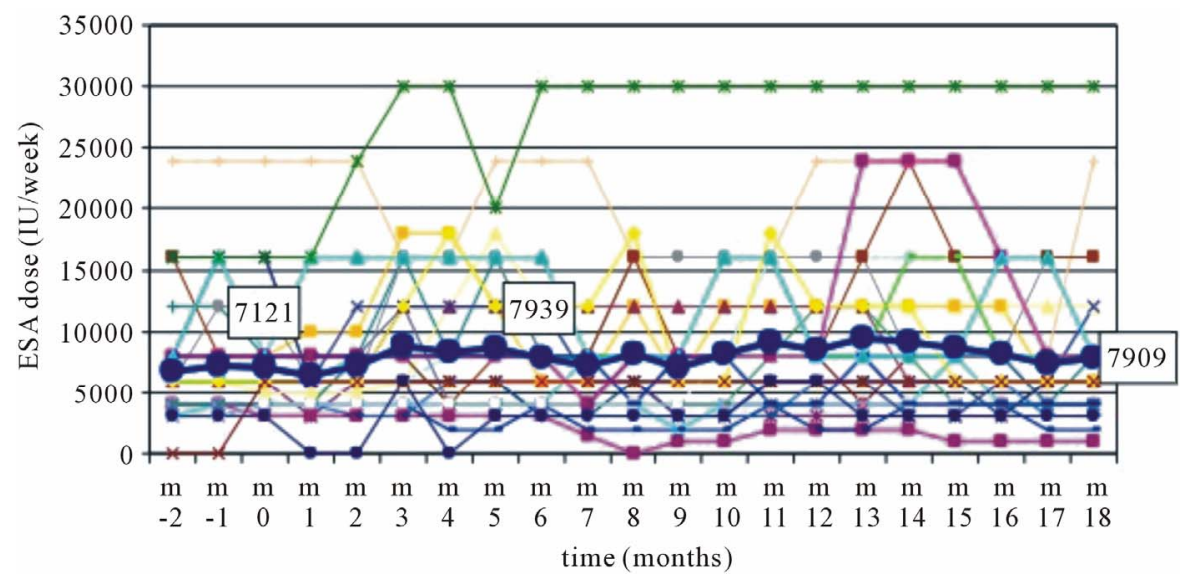

Figure 2. The weekly dose of epoetin zeta during 18 months of extended follow-up. $\mathbf{N}=33$; the bold symbols represent the mean values in $\mathrm{IU} /$ week. " $\mathrm{m}$ " = month. The mean values of the weekly dose are given in numbers when epoetin zeta was started (m 0), after 6 months (m 6) and after 18 months (m 18).

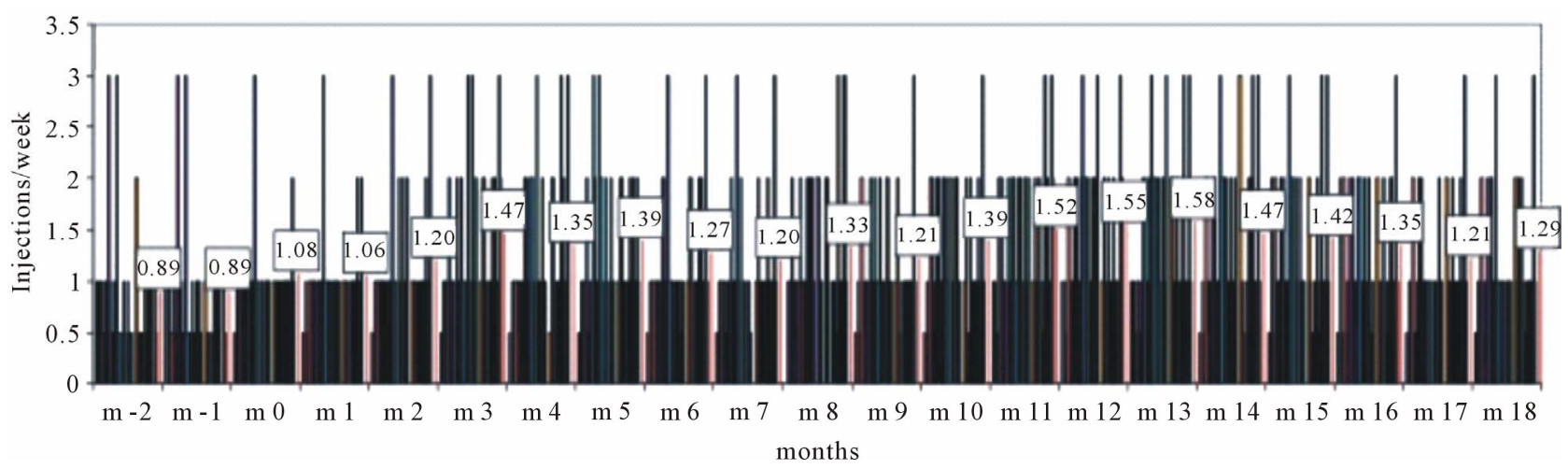

Figure 3. Frequency of ESA injections. Epoetin zeta was started in month zero $(\mathrm{m} 0)$. The mean injection frequency of $\mathbf{n}=33$ patients per month is given in numbers.

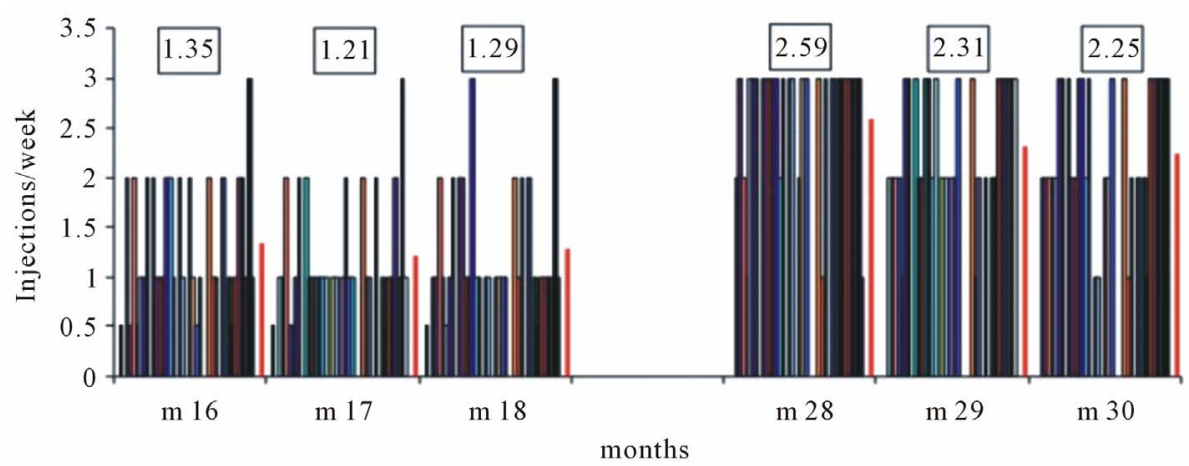

Figure 4. Frequency of epoetin zeta injections at the end of the extended follow-up period (m 18$)$ and 10 months later $(\mathrm{m} 28$ m 30). In months 19 - 21 all patients $(n=33)$ switched to 3 injections per week with dose reduction as is explained in the text. The numbers represent the mean injection frequency.

weekly dose of epoetin zeta needed to maintain stable hemoglobin levels dropped by $31.7 \%(p<0.001)$ (Figure 5).

The mean weekly dose during the end of the observation (months 28 to 30) was compared to that during the run-in phase (months -2 to 0 ) when $82 \%$ of the patients were treated with darbepoetin alpha. Figure 6 demonstrates a $21 \%$ reduction in the weekly dose of ESA ( $p=$ 0.03 ) when epoetin zeta with a mean injection frequency of 2.31/week was compared with established ESAs with a mean injection frequency of $0.89 /$ week.

No serious adverse events were observed during the 


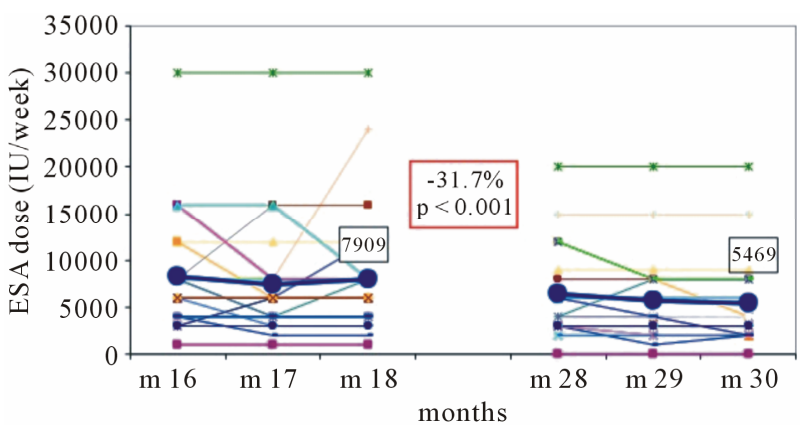

Figure 5. Weekly dose of epoetin zeta with increased injection frequency. With increased injection frequency (see Figure 4) the mean weekly dose of $n=33$ patients (bold symbols) dropped from 7909 IU/week in month 18 by $31.7 \%$ to $5469 \mathrm{IU} /$ week in month 30 (p < 0.001).

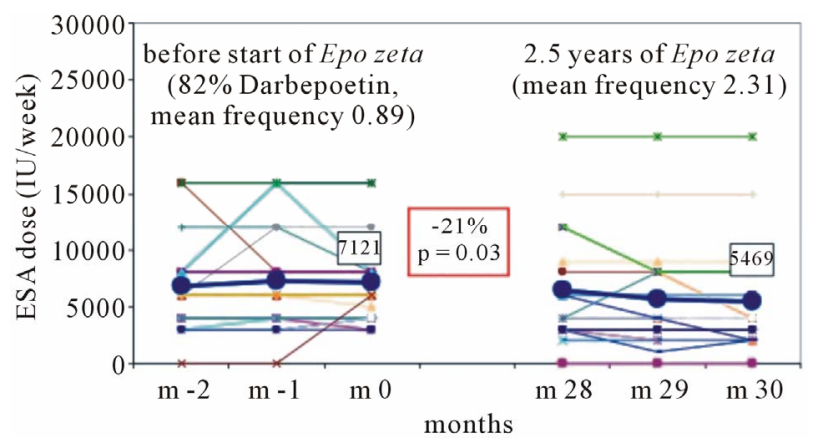

Figure 6. Weekly dose of ESAs before the start of epoetin zeta (82\% darbepoetin alpha, mean frequency 0.89 injections per week) and after 30 months of epoetin zeta with increased injection frequency (mean 2.31 injections per week). The mean weekly dose of $n=33$ patients (bold symbols) dropped from $7121 \mathrm{IU} /$ week in month 0 by $21.0 \%$ to $5469 \mathrm{IU} /$ week in month $30(p=0.03)$.

observation period, in particular there were no clinical signs that were suspicious on pure red cell aplasia.

\section{Discussion}

The present observational clinical study provides new information about the treatment of CKD patients with biosimilar epoetin zeta in two aspects: 1) during an extended follow-up of 2 and a half years epoetin zeta proved to be safe, well tolerated, and without severe adverse events. 2) Increasing the injection frequency of the short-acting epoetin zeta from 1 to 2 - 3 injections per week offers the opportunity to reduce the weekly dose significantly by approximately $31 \%$. Thus, compared to established ESAs (82\% darbepoetin alpha) the weekly dose of epoetin zeta was $21 \%$ lower $(p=0.03)$.

These surprising results are in conflict with other studies comparing the dose efficiency of short-acting epoetin alpha with that of darbepoetin alpha claiming that the weekly dose of epoetin alpha is almost twice that of darbepoetin alpha [6-9] using the same converting factor to transfer micrograms of darbepoetin alpha into I.U. of epoetin zeta ( $1 \mu \mathrm{g}=200 \mathrm{I} . \mathrm{U}$.$) .$

Differences in the biological activity and plasma half-life of the various ESAs have to be taken into account when weekly doses and a frequency of injections are prescribed. In general, the short acting epoetin zeta is recommended to be injected 3 times a week. However, our data also suggest that individualized ESA therapy is possible. Under controlled clinical conditions with hemoglobin measurements and iron supplementations on a regular basis the frequency of epoetin zeta injections can be reduced to two per week in the majority of ESRD patients and also to one per week in up to $25 \%$ of our patients on chronic intermittent hemodialysis therapy. Optimized ESA therapy should consider both the weekly dose and the frequency of injections. As a result of our clinical experience, patients on epoetin zeta should start ESA therapy with a weekly dose of approximately 100 I.U./kg/week using three injections per week. When a stable hemoglobin level of $10-12 \mathrm{~g} / \mathrm{dl}$ is established, first step of individualized adaptation should be: "keep the frequency, reduce the weekly dose". The second step then could reduce the frequency of injections from three per week to two per week and, in a few individuals to one per week, subsequently.

In conclusion, splitting the application of biosimilar epoetin zeta in three injections per week reduces the weekly dose and thereby the costs of ESA therapy of renal anemia significantly.

\section{REFERENCES}

[1] C. Baldamus, S. Krivoshiev, M. Wolf-Pflugmann, M. Siebert-Weigel, R. Koytchev and A. Bronn, "Long-Term Safety and Tolerability of Epoetin Zeta, Administered Intravenously, for Maintenance Treatment of Renal Anemia," Advances in Therapy, Vol. 25, No. 11, 2008, pp. 1215-1228. doi:10.1007/s12325-008-0111-1

[2] S. Krivoshiev, V. V. Todorov, J. Manitius, S. Czekalski, P. Scigalla and R. Koytchev, "Comparison of the Therapeutic Effects of Epoetin Zeta and Epoetin Alpha in the Correction of Renal Anaemia," Current Medical Research and Opinion, Vol. 24, No. 5, 2008, pp. 1407-1415. doi:10.1185/030079908X297402

[3] H. Schellekens, "Assessing the Bioequivalence of Biosimilars the Retacrit Case," Drug Discovery Today, Vol. 14, No. 9-10, 2009, pp. 495-499.

doi:10.1016/j.drudis.2009.02.003

[4] V. Wizemann, B. Rutkowski, C. Baldamus, P. Scigalla and R. Koytchev, "Comparison of the Therapeutic Effects of Epoetin Zeta to Epoetin Alfa in the Maintenance Phase of Renal Anaemia Treatment," Current Medical Research and Opinion, Vol. 24, No. 3, 2008, pp. 625-637. doi:10.1185/030079908X273264

[5] G. Lonnemann and E. Wrenger, "Biosimilar Epoetin Zeta in Nephrology-A Single-Dialysis Center Experience," 
Clinical Nephrology, Vol. 75, No. 1, 2011, pp. 59-62.

[6] E. Orazi, "Switch from Darbepoetin-Alpha to EpoetinAlpha: Cost and Efficacy Comparison for Haemodialytic Patients over One Year Follow-Up in a Single Centre," $G$ Ital Nefrol, Vol. 25, No. 2, 2008, pp. 223-226.

[7] F. Ranchon, S. Hedoux, M. Laville, D. Fouque, E. Decullier, F. Chapuis and L. Huot, "Direct Medical Cost of Erythropoiesis-Stimulating Agents in Anaemia Treatment of Chronic Renal Failure Patient: A Literature Review," Nephrologie \& Therapeutique, Vol. 6, No. 2, 2010, pp.
97-104. doi:10.1016/j.nephro.2009.10.004

[8] D. Goldsmith, "2009: A Requiem for rHuEPOs-But Should We Nail down the Coffin in 2010?" inical Journal of the American Society of Nephrology, Vol. 5, No. 5, 2010, pp. 929-935. doi:10.2215/CJN.09131209

[9] X. Song, S. R. Long, W. D. Marder, S. D. Sullivan and J. Kallich, "The Impact of Methodological Approach on Cost Findings in Comparison of Epoetin Alfa with Darbepoetin Alfa," Annals of Pharmacotherapy, Vol. 43, No. 7, 2009, pp. 1203-1210. doi:10.1345/aph.1L590 